\title{
Desarrollo de competencias a través de la generación y desarrollo de una idea empresarial
}

Conchado, A., Pérez, V., Fernández, E. y Egea, L.

\section{Resumen}

El presente trabajo describe el planteamiento y resultados de una actividad de enseñanza - aprendizaje en el título Grado en Ingeniería en Gestión Empresarial del centro univeresitario EDEM - Escuela de empresarios. Todos los estudiantes matriculados en el título durante el curso 2017/18 participaron en esta iniciativa. La actividad se planteó como una competición multidisciplinar de generación y desarrollo de ideas de negocio. En esta actividad se aplicaron metodologías ágiles de proyectos y técnicas de Lean Canvas. Tras finalizar la competición, los estudiantes valoraron cuantitativamente cómo la actividad había contribuído a adquirir cada una de las competencias genéricas del título. Los resultados muestran que la actividad resultó un entorno de trabajo ideal para aplicar conocimientos, tomar decisiones, trabajar en equipo y comunicación oral. Los aprendizajes adquiridos en este actividad supone una valiosa experiencia para los estudiantes, pero también para el centro y los profesores implicados en su organización. Del mismo modo, es susceptible de adaptación en otros grados de futura implantación, que incluyen proyectos transversales a nivel curricular en sus planes académicos.

Palabras clave: competencias, actividad, emprendimiento

\begin{abstract}
This paper aims to describe the conditions and learning outcomes of a teaching and learning activity in the degree BSc in Engineering and Management in the university center EDEM - Business School. All students enrolled in this degree during 2017/18 participated in this initiative. The activity was presented as a multidisciplinary competition based in the generation and development of business ideas. Agile Project management methodologies and Lean Canvas techniques were applied in this activity. Once the competition finished, students scored how the activity had contributed to the development of generic competencies inherent to the degree. Our findings show that the activity provided an adequate learning environment for the application of knowledge, decisión - making, teamwork and oral communication. These results represent a valuable experience for students, as well as for the university center ans professors. Meanwhile, this activity can be applied to other degrees including cross - sectional projects in the study plans.
\end{abstract}

Keywords: competencias, actividad, emprendimiento 


\section{Introducción}

La mentalidad emprendedora se define como la predisposición a descubrir, evaluar y explotar oportunidades de negocio. El desarrollo de esta mentalidad emprendedora implica la adquisición de determinados conocimientos y competencias orientados a la creación de nuevos negocios, como contabilidad, derecho, finanzas, gestión de equipos, marketing e incluso las habilidades de venta. No obstante, los emprendedores también desarrollan un enfoque muy particular en relación a la búsqueda de nuevas oportunidades y la tolerancia al riesgo, que pueden aplicarse a otros perfiles profesionales. Como exponen McGrath and MacMillan (2000), los emprendedores se caracterizan por un estado de búsqueda permanente de nuevas oportunidades, que persiguen con disciplina. Este esfuerzo lo dedican solo a las mejores oportunidades y se centran en la ejecución de las ideas de negocio. Para ello, son capaces de comprometer a su equipo de trabajo, o sus recursos disponibles, para que den lo mejor de sí mismos en cada proyecto.

Por el contrario, la educación de los futuros ingenieros en la actualidad está fundamentada en los conocimientos científico - técnicos clálicos que constituyen la base de las tecnologías actuales. Este aprendizaje de los fundamentos matemático - físicos es clave en su formación ya que les permitirá posteriormente modelizar y resolver problemas en el entorno de los procesos industriales y empresariales en los que desarrollen su carrera profesional. No obstante, el rápido desarrollo tecnológico que se ha producido en los últimos años implica que los futuros ingenieros no sólo tendrán que ser capaces de resolver problemas ya existentes, sino de aportar valor y nuevas soluciones en las empresas donde trabajen. La generación de valor no está limitada a la creación de nuevas empresas y el desarrollo de ideas de negocio. Independientemente del área de especialización, los futuros graduados en ingeniería deberán ser capaces de facilitar el cambio de la empresa hacia nuevas tecnologías que les ayuden a satisfacer las necesidades de sus clientes, de una forma cada vez más eficiente e innovadora. Al igual que la dirección de empresas resulta una disciplina en sí misma, también existen determinados perfiles de profesionales especialistas que son capaces de liderar tecnológicamente a la empresa hacia el éxito (Drucker, 1986). Así, el intraemprendimiento constituye hoy en día una forma de emprendimiento para garantizar la supervivencia y desarrollo económico de las empresas (Trujillo, 2008).

Del mismo modo, si queremos ofrecer productos de calidad, debemos ser capaces de diseñar y producir aquello que el cliente necesita. La educación superior en ingeniería facilita la evaluación de proyectos en el ámbito del diseño, la producción, e incluso entre ambos. Pero debemos ser conscientes de que trabajar en estas dimensiones, olvidando las necesidades de cliente, puede conducirnos a una zona de satifacción exclusivamente industrial. Por ello, debemos movernos hacia la intersección de las tres dimensiones, con el fin de garantizar la satisfacción plena del cliente.

(cc) EY-NC-ND 2018, Universitat Politècnica de València

Congreso IN-RED (2018) 
Así, determinados enfoques de la mentalidad emprendedora pueden orientar y guíar a los ingenieros en el desarrollo de actividades de intraemprendimiento. Los conocimientos y competencias relacionadas con emprendimiento resultan muy recomendables para todos los estudiantes de ingeniería. No debería plantearse como una asignatura optativa reservada a determinados estudiantes con motivación personal por emprender (Bosman and Fernhaber, 2018). El principal factor motivador en la intención emprendedora de los estudiantes de ingeniería es la necesidad de autonomía en la organización y gestión de su propio trabajo (Barba-Sánchez \& Atienza-Sahuquillo, 2018). No obstante, debemos preguntarnos cuál es la forma más efectiva de adquirir este aprendizaje. Neck et al. (2014) afirman que para aprender emprendimiento, es necesario emprender. Pero cualquier experiencia práctica en emprendimiento debe estar siempre basada en una metodología que la soporte con el fin de lograr un aprendizaje eficiente.

En este proceso de diseñar un nuevo producto, o mejorar un producto o servicio ya existente, los profesionales hoy en día trabajan por proyectos. Durante esta fase de desarrollo, se aplican metodologías clásicas de gestión de proyectos inicialmente creadas para gestionar proyectos de construcción, obra civil e incluso militares. Esta forma de trabajo parte del supuesto de que el trabajo puede compartirmentarse, de modo que la ejecución del trabajo se organiza en diversos paquetes de trabajo secuenciales. No obstante, los equipos de trabajo actuales deben enfrentarse continuamente a cambios en las especificaciones y requisitos del cliente, la tecnología disponible e incluso la propia composición de los miembros del equipo. Las metodologías ágiles se han diseñado en base al supuesto de que las condiciones de trabajo pueden variar y pretenden facilitar la respuesta y adaptación a estos cambios (Ma, y Morris, 2017).

Por todo ello, las instituciones de educación superior se enfrentan al reto de formar a sus estudiantes en ingeniería en los conocimientos y competencias específicas que necesitarán en sus puestos de trabajo, al tiempo que les forman en competencias genéricas. En los últimos años, la Universidad Politécnica de Valencia está trabajando para introducir nuevas actividades de enseñanza - aprendizaje en cada asignatura, orientadas a la evaluación de competencias genéricas, en especial en las consideradas puntos de control. No obstante, cabe preguntarse en qué medida una actividad transversal y coordinada entre varias asignaturas puede contribuir al desarrollo de estas competencias genéricas. El planteamiento de retos reales y multidisciplinares, como la generación y desarrollo de ideas empresariales, puede ser el escenario perfecto para motivar a los estudiantes. El presente trabajo aborda esta cuestión y muestra los resultados obtenidos a través de la actividad en el Grado en Ingeniería en Gestión Empresarial durante el curso 2017/18. 


\section{Objetivos}

Con el fin de dar respuesta a las cuestiones planteadas anteriormente, el presente trabajo pretende analizar las competencias adquiridas por una actividad extracurricular destinado a desarrollar competencias genéricas entre los estudiantes de ingeniería en el título de Grado en Ingeniería en Gestión Empresarial. Para ello, se plantean los siguientes objetivos específicos:

1. Evaluar el grado de adquisición de competencias del título mediante esta actividad

2. Valorar las aportaciones cualitativas de los participantes como puntos fuertes y áreas de mejora

\section{Desarrollo de la innovación}

\subsection{El Grado en Ingeniería en Gestión Empresarial}

El Centro Universitario EDEM, adscrito a la Universitat de València y a la Universitat Politècnica de València, es una institución académica promovida por la Escuela de Empresarios, Fundación de la Comunitat Valenciana (EDEM) en la que se imparten estudios universitarios de carácter oficial de Grado y títulos propios de Postgrado.

En este centro se imparte el Grado en Ingeniería y Gestión Empresarial, titulación oficial adscrita a la Universitat Politècnica de València, que comenzó su implantación en 2014 con la voluntad de formar a los ingenieros del siglo XXI en conocimientos y habilidades fundamentales para la empresa, para que ocupen posiciones de gestión empresarial con orientación técnica en proyectos globales o como intraemprendedores, desarrollando nuevas áreas de negocio en una empresa.

\subsection{Participantes}

Todos los estudiantes matriculados en el Grado en Ingeniería y Gestión Empresarial durante el curso 2017/18 participaron en la actividad, alcanzando un número global de 90 participantes en la actividad. Debido a que el título se encuentra en el tercer año de implantación, estos 90 estaban matriculados en $1^{\circ}, 2^{\circ}$ y $3^{\circ}$ curso.

Cada equipo estaba formado por 5 personas, de modo que el centro formó 18 equipos. No se permitió la formación de equipos a criterio de los estudiantes. En la composición de los equipos se empleó como criterio fomentar la heterogeneidad entre los miembros. De este modo, en cada equipo se asignó al menos un estudiante de $3^{\circ}$ curso y otro de $2^{\circ}$ curso. No obstante, la actividad fue valorada por 77 estudiantes, obteniendo una tasa de respuesta del $85.5 \%$. 


\subsection{Breve descripción de la actividad}

La actividad tuvo una duración de cuatro días de trabajo. Tras una presentación inicial, los estudiantes trabajaron de forma autónoma en equipo para desarrollar una idea de negocio. Todos los equipos debían proponer un producto físico, que cumpliera la normativa legal, que fuera rentable y conforme a cuestiones éticas. Para ello, se organizaron varias sesiones de trabajo coordinadas por los profesores de distintas materias del título. El objetivo de estas sesiones era organizar el trabajo en la elaboración de sucesivos elementos entregables, que guiaban a los equipos hacia el desarrollo de la idea de negocio y la construcción del prototipo. En dichas sesiones, los profesores adaptaron los objetivos a los conocimientos de sus asignaturas, de modo que fuera posible avanzar en el desarrollo de la actividad, por medio de la aplicación práctica de los contenidos del título.

En el desarrollo de la actividad aplicamos la metodología Sprint, creada y desarrollada por Google Ventures (Knapp, 2013). Esta metodología proporciona un marco de trabajo estructurado en cinco fases que permite abordar problemas estratégicos a través de técnicas de prototipado rápido y validación de producto. Desde su creación en el año 2010, se ha aplicado en múltiples entornos como plataformas tecnológicas, por ejemplo destinadas al bienestar de personas mayores (Keijzer-Broers y De Reuver, 2016) o aplicaciones web para el análisis de datos meteorológicos (Grahl, Niklas y Söderling, 2015).

Asimismo, se ha combinado esta metodología con la técnica del Lean Canvas, para la generación y diseño de ideas de negocio. Constituye una adaptación del modelo Canvas y se centra en la identificación de problemas y necesidades del cliente enfocada a la propuesta de soluciones y métricas adaptadas. Con esta información es posible generar una propuesta de valor única, que constituye el valor diferencial y motivador para que los consumidores seleccionen nuestro producto (Maurya, 2012). Esta combinación de metodologías no es novedosa y ya se ha empleado en otros entornos, principalmente relacionados con el desarrollo de software (Nidagundi y Novickis, 2017).

Esta idea debía convertirse en un prototipo preliminar que posteriormente validaron con usuarios potenciales. Para ello se dirigieron a estudiantes matriculados en otros títulos, personal del centro y otros posibles usuarios. Con las mejoras recopiladas en esta sesión de testeo, propusieron una version final, que fue evaluada en sesión pública. Las valoraciones de cada equipo se calcularon a través de una ponderación del trabajo elaborado a lo largo de la actividad, las valoraciones de los compañeros y las valoraciones del tribunal. Asimismo, al finalizar la actividad, los estudiantes valoraron mediante una escala Likert de 1 a 5 en qué medida la actividad había contribuido al aprendizaje de las competencias genéricas (básicas y generales) del título.

\subsection{Recogida y análisis de datos}

Tras la presentación de las ideas de negocio y prototipos desarrollados por los estudiantes, los estudiantes valoraron su nivel de satisfacción con la actividad y el grado en que la actividad les había ayudado a desarrollar las competencias del título. Al finalizar, se elaboró un ranking de puntuaciones y se reconoció públicamente el valor de los equipos en 
las primeras posiciones. Estos resultados no tuvieron repercusión académica en calificaciones o implicación de los estudiantes en otras actividades. Para lograr la máxima implicación de los estudiantes, se generó un clima de competición empresarial que supone la aplicación de técnicas de gamificación en el aula (Zichermann y Linder, 2013). No obstante, la recopilación de datos sobre la valoración de competencias se realizó a la semana siguiente, tras la finalización de la actividad. Las valoraciones se recogieron de forma individual, sobre el listado de competencias del título impreso en papel. El momento de recogida de datos fue antes del comienzo de la primera sesión lectiva. Para valorar el nivel de contribución de la actividad a cada competencia se empleó una escala Likert desde 1 (Nada) hasta 5 (Mucho). La selección de competencias a evaluar fue extraída del listado de competencias generales de la memoria de verificación del título.

Asimismo, se solicitó a los estudiantes que valoraran con un enfoque cualitativo tres puntos fuertes y otros tres puntos débiles de la activdad. Finalmente se puso a disposición de los estudiantes un campo abierto de comentarios. A la hora de examinar los resultados en este apartado de la investigación, se registraron todas las aportaciones con contenido novedoso. Además, se contabilizó el número de repeticiones (frecuencias) de comentarios relativos a aportaciones anteriores. Así, fue posible trazar un análisis aproximado al histograma con el número de estudiantes que señalaron cada aportación cualitativa.

\section{Resultados}

Con el fin de abordar los objetivos planteados anteriormente, la siguiente sección expone los resultados de aprendizaje de la actividad. En particular, el análisis de valoraciones cuantitativas permitirá evaluar el grado de adquisición de las competencias del título, extraídas de la memoria de verificación, a través de la actividad.

Los resultados muestran que la actividad ha contribuido al aprendizaje de cómo aplicar conocimientos de forma profesional, transmitir información y analizar distintos elementos para la toma de decisiones empresariales. También ha fomentado que los estudiantes aprendan a reunir e interpretar datos y trabajar en equipo. Si bien los estudiantes aprenden en el título herramientas específicas para la toma de decisiones, en este trabajo se han enfrentado al reto de resolver un problema problema y asumir las consecuencias de sus decisiones.

En términos generales, es fácil observar que las competencias más relacionadas con la actividad son las de carácter genérico. Por el contrario, las competencias específicas como técnicas, habilidades y herramientas tecnológicas y económicas, expresarse en lenguajes formales, o problemas técnicos complejos obtienen los valores mínimos. Estos resultados están en línea con el planteamiento de la actividad, de carácter transversal al título. En particular, únicamente se solicitó a los estudiantes que redactaran un informe sobre las conclusiones de la investigación de mercado relativa a su idea de negocio. Por ello, no resulta sorprendente que la capacidad de expresarse en lenguajes formales haya obtenido este valor.

(c) ) EY-NC-ND 2018, Universitat Politècnica de València

Congreso IN-RED (2018) 


\begin{tabular}{lcc}
\hline Descripción competencias & Media & Desv \\
\hline $\begin{array}{l}\text { Aplicar conocimientos al trabajo de forma profesional, elaborar y defender } \\
\text { argumentos y resolver problemas }\end{array}$ & 4.03 & 0.76 \\
$\begin{array}{l}\text { Transmitir información, ideas, problemas y soluciones a un público tanto } \\
\text { especializado como no especializado }\end{array}$ & 3.99 & 0.80 \\
$\begin{array}{l}\text { Aprender a analizar los diferentes elementos que interactúan en la toma de } \\
\text { decisiones empresariales }\end{array}$ & 3.96 & 0.85 \\
$\begin{array}{l}\text { Capacidad para tomar decisiones en ambientes de certidumbre e incertidumbre } \\
\text { empresarial }\end{array}$ & 3.93 & 0.90 \\
$\begin{array}{l}\text { Reunir e interpretar datos relevantes para emitir juicios que incluyan una reflexión } \\
\text { sobre temas relevantes de índole social, científica o ética }\end{array}$ & 3.85 & 0.80 \\
$\begin{array}{l}\text { Capacidad para trabajar en equipo en entornos multilingües, multidisciplinares y } \\
\text { multiculturales }\end{array}$ & 3.82 & 1.02 \\
$\begin{array}{l}\text { Definir, resolver y exponer de forma sistémica problemas técnicos complejos } \\
\begin{array}{l}\text { Desarrollar habilidades de aprendizaje necesarias para emprender estudios } \\
\text { posteriores con alto grado de autonomía }\end{array}\end{array}$ & 3.65 & 0.74 \\
$\begin{array}{l}\text { Saber expresarse en lenguajes formales, gráficos y simbólicos necesarios para } \\
\text { entenderse en ambientes ingenieriles y empresariales }\end{array}$ & 3.62 & 0.95 \\
$\begin{array}{l}\text { Usar las técnicas, habilidades y herramientas tecnológicas y económicas, necesarias } \\
\text { para la práctica profesional de la ingeniería y gestión empresarial }\end{array}$ & 3.61 & 0.92 \\
\hline
\end{tabular}

Tabla 1. Estadísticos descriptivos de adquisición de competencias

Con el fin de enriquecer la información y conclusiones anteriores, a continuación se exponen las valoraciones cualitativas y comentarios realizados por los estudiantes. Algunos estudiantes señalaron que el tiempo estimado para el desarrollo de las actividades era insuficiente y afirmaron que les hubiera gustado tener más autonomía para gestionar su trabajo. Por el contrario, la mayoría de los estudiantes reconocieron que habían mejorado su capacidad de organización gracias a esta actividad. Asimismo, muchos estudiantes indicaron que habían mejorado sus habilidades de trabajo en equipo a través de la colaboración entre estudiantes de distintos cursos. Finalmente, también destacaron que habían mejorado sus competencias de comunicación oral, pues la presentación final ante la audiencia era obligatoria para todos los estudiantes.

\section{Conclusiones}

La actividad del Grado en Ingeniería y Gestión Empresarial del Centro Universitario EDEM ha constituido una actividad de innovación docente exitosa en la adquisición de competencias genéricas. Los estudiantes se han implicado por completo en el desarrollo de su idea de negocio y construcción del prototipo, a pesar de que los resultados no tenían implicaciones académicas en calificaciones o participación en otras actividades relacionadas con el título. El desarrollo de esta actividad ha implicado la combinación de distintas herramientas para la innovación educativa, como el aprendizaje

(c) ) EY-NC-ND 2018, Universitat Politècnica de València

Congreso IN-RED (2018) 
orientado a proyectos o las técnicas de gamificación, sin perder la orientación hacia la aplicación práctica de los conocimientos adquiridos en el aula.

Esta actividad supone un valioso aprendizaje para los estudiantes, al tener la oportunidad de desarrollar su propia idea de negocio, en un entorno controlado y guíado por sus profesores. En relación al primer objetivo del trabajo, el análisis cuantitativo del nivel de adquisición de competencias muestra que contribuye al desarrollo de competencias genéricas como entorno de trabajo ideal para aplicar conocimientos, tomar decisiones, trabajar en equipo y comunicación oral. Estos resultados están en línea con el planteamiento de la actividad, de carácter transversal al título y fueron confirmados por las valoraciones cualitativas de los estudiantes, en base al segundo objetivo del trabajo. Así, el análisis de los comentarios de los alumnos permitió confirmar la actividad es un medio de aprendizaje eficaz para las habilidades de trabajo en equipo y comunicación oral. Del mismo modo, las respuestas de los estudiantes permitió identificar la capacidad de organización y gestión de tiempo como un nuevo resultado de aprendizaje vinculado a esta actividad.

Al mismo tiempo, se ha confirmado que este tipo de innovaciones docentes contribuye al desarrollo de competencias genéricas, mientras que puede asignarse al contexto de cada asignatura la enseñanza y aprendizaje de las competencias específicas. Además, esta experiencia constituye un punto de partida para identificar a los estudiantes con orientación emprendedora, que en el futuro podrían desarrollar su propio proyecto empresiarial aprovechando los recursos. Esta actividad es susceptible de aplicación en otros grados de futura implantación, que incluyen proyectos transversales a nivel curricular en sus planes académicos. No obstante, cabe destacar que requiere un esfuerzo considerable de organización y coordinación académica por parte de los profesores implicados. Del mismo modo, el planteamiento de la actividad debe considerar el momento en que se encuentran los estudiantes a nivel curricular. No todos los estudiantes habrán estudiado los conocimientos del curso completo en que estén matriculados, y la actividad debe ajustarse a los contenidos vistos en el momento de la ejecución. En la aplicación de dicha actividad a otros títulos pueden plantearse líneas futuras de trabajo, como la generación y desarrollo de ideas basadas en problemas reales planteados por empresas. E incluso la composición de equipos de trabajo formados por alumnos de distintos títulos, que enriquecerían el enfoque multidisciplinar de las soluciones propuestas por el equipo.

\section{Referencias}

Barba-Sánchez, V., \& Atienza-Sahuquillo, C. (2018). Entrepreneurial intention among engineering students: The role of entrepreneurship education. European Research on Management and Business Economics, 24(1), 53-61 
Bosman, S. F. (2018). Teaching the Entrepreneurial Mindset to Engineers. New York, NY: Springer International Publishing.

Drucker, P. (1986). La economía empresaria innovadora (prólogo). En La innovación y el empresario innovador (pp. 11-29). Bogotá: Norma.

Grahl, N., \& Söderling, A. (2015). Design and implementation of a web interface for Axis global testing Live Sites. Ergonomics and Aerosol Technology. LUP Student Papers. Lund: Lund University Libraries.

Keijzer-Broers, W. J., \& de Reuver, M. (2016). Applying Agile Design Sprint Methods in Action Design Research: Prototyping a Health and Wellbeing Platform. In International Conference on Design Science Research in Information Systems (pp. 6880). Springer, Cham.

Knapp, J. (2013). How To Conduct Your Own Google Ventures Design Sprint. Fast Company, 24.

Ma, M., \& Morris, L. (2017). The Agile Innovation Sprint. International Management Review, 13(1), 92.

Maurya, A. (2012). Running lean: iterate from plan A to a plan that works. O'Reilly Media, Inc. UNIR Universidad de La Rioja.

McGrath, R. G., \& MacMillan, I. C. (2000). The entrepreneurial mindset: Strategies for continuously creating opportunity in an age of uncertainty. Harvard Business Press.

Neck, H. M., Greene, P. G., \& Brush, C. G. (Eds.). (2014). Teaching entrepreneurship: A practice-based approach. Edward Elgar Publishing.

Nidagundi, P., \& Novickis, L. (2017 Introducing lean canvas model adaptation in the scrum software testing. Procedia Computer Science, 104, 97-103.

Trujillo, M.-A. \& Guzmán, A. (2008). Intra/Entrepreneurship: A Review of the Theoretical Construct, its Implications, and an Agenda for Future Research. Cuadernos de Administración, Vol. 21, No. 35, pp. 37-63.

Zichermann, G., \& Linder, J. (2013). The gamification revolution: How leaders leverage game mechanics to crush the competition. New York: McGraw-Hill. 